\section{Celebrating 40 years of progress in bone marrow transplantation: a report from the 40th Annual Meeting of the European Society for Blood and Marrow Transplantation}

\author{
Livio Pagano' \& Sue Lyon ${ }^{* 2}$

\section{0th Annual Meeting of the European Society for Blood and Marrow Transplantation, Milan, Italy, 30 March-2 April 2014}

\begin{abstract}
The European Society for Blood and Marrow Transplantation was established in 1974 to enable scientists and physicians involved in clinical bone marrow transplantation to share their experience and develop cooperative studies. The organization celebrated its 40th anniversary with a meeting that considered hematopoietic stem cell transplantation not as a standalone procedure, but as part of a complex therapeutic program managed by a multidisciplinary professional team. The role of antifungal prophylaxis, emerging resistance in Aspergillus, the management of mucormycosis and new guidelines on antifungal therapy were among the topics discussed by the physicians, nurses, allied health professionals and scientists attending the 40th Annual Meeting of the European Society for Blood and Marrow Transplantation.
\end{abstract}

As a scientific society, the European Society for Blood and Marrow Transplantation (EBMT) has grown in parallel with use of hematopoietic stem cell transplantation (HSCT) - the millionth bone marrow transplant was reported in early 2013 - and now has members in over 500 centers worldwide. This global expansion of EBMT has been built on a foundation of basic and clinical research, including both randomized clinical trials and observational studies. According to Catherine Cordonnier (Créteil, France), observational or noninterventional studies do not replace gold standard, prospective clinical trials: "Knowing what people do does not tell us what we ought to do."

Observational studies can, however, complement clinical trials if they are well designed and have a clearly stated plan for statistical analysis. In particular, observational studies are often the only credible approach for rare diseases. These studies may also identify epidemiological trends and other important issues that may in turn help to improve management in the clinic and provide hypotheses for future clinical trials. As examples, Cordonnier cited the emergence of invasive aspergillosis in patients with chronic lymphoproliferative disorders identified by the Surveillance des Aspergilloses Invasives en France (SAIF) network [1] and the variability in the incidence and species distribution of candidemia seen in the seven countries included in the Latin American Invasive Mycosis Network [2].

Most HSCT continue to be performed in Europe and the Americas, with the east Mediterranean/ Africa region representing less than 2\% of global HSCT [3]. During EMBT 2014, an observational study from the Nigerian Group for Blood and Marrow Transplant reported outcomes of their first three successful HSCTs, all in patients with sickle cell disease.

\section{KEYWORDS}

- antifungal prophylaxis

- antifungal resistance

- Aspergillus $\bullet$ bone marrow transplantation

- candidemia • ECIL-5

- hematopoietic stem

cell transplantation

- invasive fungal infection

- mucormycosis 
The first transplant was performed in September 2011 for a 7-year-old patient with a cerebrovascular accident, followed in August 2012 by HSCT in a 12-year old with recurrent vaso-occlusive crisis, who received a re-transplant in May 2013. The third HSCT in July 2013 was for a 15-year patient with recurrent vaso-occlusive crisis. All three patients had allogeneic HSCT from identical siblings. There was no recorded acute or chronic graft-versus-host disease. Chimerism for the first HSCT patient was $95 \%$ at 2 years post-HSCT, $0 \%$ for the second patient after the retransplant and $57 \%$ at day 27+ after HSCT for the third patient [4].

The Nigerian Group for Blood and Marrow Transplant was established in 2013 to coordinate and regulate HSCT in Nigeria. Other recent developments in that country include a bone marrow registry at the University of Nigeria Teaching Hospital (Enugu, Nigeria) to increase the potential for unrelated HSCT, and an HLA laboratory at the Obafemi Awolowo University Teaching Hospital (Ile-Ife, Nigeria).

\section{Trials into practice}

Observational studies can also be important in confirming that findings from clinical trials can be translated into routine clinical practice. As an independent investigator-led group of Italian hematologists, SEIFEM has performed several retrospective observational studies to investigate the epidemiology of fungal infections in patients with hematological malignancies and following HSCT [5-7]. Based on this experience, the group has recently undertaken a prospective, observational study to evaluate the efficacy in clinical practice of antifungal prophylaxis with posaconazole and itraconazole during the first induction after remission of acute myeloid leukemia. The 1192 consecutive patients were recruited from the 31 participating Italian centers and were included in the SEIFEM 2010-A PR registry [8].

Livio Pagano (Rome, Italy) reported that, of the 887 patients who received intensive chemotherapy and who were included in the analysis, 214 (24\%) developed an invasive fungal infection (IFI; 73 [8.2\%] proven/probable; 53 moulds and 20 yeasts). On multivariate analysis, there was no association between IFI and the patient's hobbies, area of residence, house type, pets, fireplace use, alcohol consumption, or use of illicit drugs. There was, however, a significant association between the development of IFI and several prehospital risk factors, including the patient's age, performance status, smoking history, body weight and the presence of diarrhea and esophagitis. In future, SEIFEM hopes to validate these risk factors in other hematologypatient populations in order to design a risk score to identify patients who may need earlier antifungal prophylaxis [8].

By identifying a significant association between posaconazole prophylaxis and a reduced risk of IFI, SEIFEM 2010-A PR confirmed previous observational findings from SEIFEM 2010-B registry. This registry included 576 patients, 515 of whom were receiving systemic prophylaxis. Although the patients' baseline clinical characteristics were similar, patients receiving prophylaxis with posaconazole had a significantly lower risk of breakthrough IFI than those given itraconazole (18.9 vs $38.7 \%$, $\mathrm{p}<0.001)$. The posaconazole group also had a lower risk of proven/probable mould infections (2.7 vs $10.7 \%$ for itraconazole, $\mathrm{p}=0.02$ ). Pagano commented that the low rate of proven-probable mould infections in the posaconzole group was not only similar to that reported in the pivotal clinical trials $[9,10]$, but also resulted in a significant reduction in the use of frontline antifungal therapy [11].

\section{Resistance \& prophylaxis}

Triazole drugs have been a mainstay of the treatment of IFI since the introduction of fluconazole in early 1990, but there are now concerns about the emergence of resistant strains of Aspergillus. According to Paul Verweij (Nijmegen, The Netherlands), there are two routes that may result in triazole resistance. The first is the patient route, when resistance develops following longterm antifungal therapy for chronic Aspergillus disease. In the second, the environmental route, a triazole-naïve patient is infected by spores that have previously developed resistance following exposure to agricultural and industrial triazoles that are very similar to the antifungal agents used in human healthcare [12].

The potential for the development of multidrug-resistant strains of Aspergillus fumigatus raises the possibility that antifungal prophylaxis with a triazole may become less effective. There has been one reported case of culture-proven invasive aspergillosis under posaconazole prophylaxis caused by triazole-resistant $A$. fumigatus bearing the TR34L98H mutation, the predominant environmental resistance mechanism [13]. Clinical data are otherwise lacking, and animal 
models suggest that in neutropenic patients posaconazole remains effective in preventing invasive aspergillosis due to resistant isolates, retaining some activity at a minimum inhibitory concentration of $>16$ [14]. Verweij suggested that one explanation for this continuing effectiveness might be the lower fungal burden seen under prophylaxis, but considered it to be probably more important that posaconazole concentrations are 20-30 times higher in the lung at the site of the infection than in the blood [15].

\section{Mucormycosis}

In hemato-oncology patients receiving antifungal prophylaxis, there is some evidence of a shift to non-Aspergillus species, especially to Mucorales [16]. Specific Mucorales strains such as Cunninghamella may be becoming more frequent [17] but reported cases of mucormycosis generally remain rare in these patients [18]. However, Pagano considered that the reported rate of infection might be artificially low due to the difficulties in diagnosing mucormycosis. Conventional microbiological testing has low sensitivity, and radiological signs are similar to those of aspergillosis, though the reversed halo sign on computed tomography might be helpful in diagnosing mucormycosis in neutropenic leukemia patients [19].

Despite changing epidemiology and diagnostic challenges, mortality due to Mucorales has been falling in hemato-oncology patients [20]. According to Pagano, this is due to the effectiveness of current treatment approaches that combine underlying disease control, surgical debridement when feasible and antifungal therapy [21]. However, he emphasized that prompt initiation of therapy is essential when managing mucormycosis, given the doubling of mortality (from 48.6 to $82.8 \%$ ) if antifungal therapy is started $\geq 6$ days after diagnosis [22].

\section{European Conference on Infections in Leukemia: new recommendations for antifungal therapy}

Management of mucormycosis was among the topics discussed by Cordonnier during her presentation of the latest guidelines from the European Conference on Infections in Leukemia (ECIL) on antifungal therapy of IFI in hemato-oncology patients. She explained that the goals of these revised guidelines (ECIL-5) [23] were to update recommendations based on analysis of new data for Candida, Aspergillus and
Mucorales infections in hematological patients, and to replace the five-level, A-E scale used for strength of recommendations in the last Candida and Aspergillus guidelines with the three-level, A-C scale used previously for Mucorales. In this new scale, grade A denotes recommendations that are supported by good evidence, grade B moderate evidence, while grade $\mathrm{C}$ indicates poor evidence to support a recommendation for use.

Given the difficulties in diagnosing Mucorales infections, ECIL reviewed current evidence to support novel diagnostic methods such as Mucorales-specific T-cell detection, molecular tests and matrix-assisted laser desorption/ionization. Although promising, these approaches require further investigation before they can be given a graded recommendation. There continue to be no validated minimum inhibitory concentration breakpoints to determine susceptibility categories for any drug used in the treatment of mucormycosis.

Amphotericin B lipid complex (ABLC) and liposomal amphoterin $\mathrm{B}(\mathrm{LAmB})$ are still first line in mucormycosis, with a grade B recommendation. Evidence for first-line use of posaconazole and combination treatment remains poor (grade C). In contrast, posaconazole retains its grade $\mathrm{B}$ recommendation in salvage therapy, and there is now a grade $B$ recommendation to support use of the combinations of LAmB and caspofungin or LAmB and posaconazole. The iron-chelating drug deferasirox is not recommended in salvage therapy. There is a new recommendation (grade B) for posaconazole in maintenance therapy if the patient has previously responded, is intolerant to first-line therapy or has stable disease.

When treating candidemia in hematology patients, ECIL considers that, before species identification, there is now good evidence for a grade $A$ recommendation for the echinocandins and LAmB. Following species identification, echinocandins are first line for Candida albicans, Candida glabrata and Candida krusei, with fluconazole reserved for Candida parapsilosis. Removal of the central venous catheter has a grade B recommendation in hematology patients, and treatment with an echinocandin or LAmB is preferred if the catheter cannot be removed.

In invasive aspergillosis, voriconazole remains first line (grade A), followed by grade B recommendations for LAmB and then ABLC. Caspofungin, itraconazole, amphotericin B 
colloidal dispersion (ABCD), voriconazole plus anidulafugin have grade $\mathrm{C}$ recommendations. Other combinations also have grade $\mathrm{C}$ recommendations and amphotericin $\mathrm{B}$ deoxycholate is not recommended. Since there are no new data for the salvage therapy of invasive aspergillosis, LAmB, ABLC, caspofungin and posaconazole retain their grade $B$ recommendations, although there is no evidence for their use in voriconazole failure. There is now evidence to support a grade $B$ recommendation for combination therapy.

Since its first meeting in 2005, ECIL has been at the forefront of developing evidence-based clinical guidelines. It continues to be organized under the auspices of the EBMT, together with the Infectious Diseases Group of the European Organisation for Research and Treatment of Cancer, the Supportive Care Group of the European LeukemiaNet and the International Immunocompromised Host Society, all organizations that share the goal of understanding and improving the management of patients at risk of infectious complications following treatment for leukemia or HSCT.

\section{Financial \& competing interests disclosure} $S$ Lyon's attendance at EBMT and her contribution to the writing of this report were supported by MSD. The authors have no other relevant affiliations or financial involvement with any organization or entity with a financial interest in or financial involvement in the subject matter or materials discussed in the manuscript apart from those disclosed.

No writing assistance was utilized in the production of this manuscript.

\section{References}

1 Lortholary O, Gangneux JP, Sitbon K et al. Epidemiological trends in invasive aspergillosis in France: the SAIF network (2005-2007). Clin. Microbiol. Infect. 17(12), 1882-1889 (2011).

2 Nucci M, Queiroz-Telles F, Alvarado-Mature $\mathrm{T}$ et al. Epidemiology of candidemia in Latin America: a laboratory-based survey. PLoS ONE 6(8), e59373 (2013).

3 WBMT (Worldwide Network for Blood and Marrow Transplantation). 1 millionth blood stem cell transplant marks major medical milestone.

www.wbmt.org

4 Bazuaye NG, Enomamien B. Hematopoietic stem cell transplantation (HSCT) for hemoglobinopathy (sickle cell disease) in Nigeria - 2013 update by Nigerian Group for Blood and Marrow Transplant (NGBMT). Bone Marrow Trans. 49(Suppl. 1), PH-P459 (2014).

5 Pagano L, Caira M, Candoni A et al. The epidemiology of fungal infections in patients with hematologic malignancies: the SEIFEM-2004 study. Haematologica 91(8), 1068-1075 (2006).

6 Pagano L, Caira M, Nosari A et al. Fungal infections in recipients of hematopoietic stem cell transplants: results of the SEIFEM B-2004 study - Sorveglianza Epidemiologica Infezioni Fungine Nelle Emopatie Maligne. Clin. Infect. Dis. 45(9), 1161-1170 (2007).

7 Pagano L, Caira M, Candoni A et al. Invasive aspergillosis in patients with acute myeloid leukemia: a SEIFEM-2008 registry study. Haematologica 95 (4), 644-650 (2010).

8 Caira M, Candoni A, Busca L et al. Pre-hospital risk factors for invasive fungal disease in newly diagnosed acute myeloid leukaemia: final results from SEIFEM 2010-A PR. Presented at: 23rd European Congress on Clinical Microbiology and Infectious Diseases (ECCMID). Berlin, Germany, 30 April 2013.

9 Cornely OA, Maertens J, Winston DJ et al. Posaconazole vs. fluconazole or itraconazole prophylaxis in patients with neutropenia. N. Engl. J. Med. 356(4), 348-359 (2007).

10 Ullmann AJ, Lipton JH, Vesole DH et al. Posaconazole or fluconazole for prophylaxis in severe graft-versus-host disease. N. Engl. J. Med. 356(4), 335-347 (2007).

11 Pagano L, Caira M, Candoni A et al. Evaluation of the practice of antifungal prophylaxis use in patients with newly diagnosed acute myeloid leukemia: results from the SEIFEM 2010-B registry. Clin. Infect. Dis. 55(11), 1515-1521 (2012).

12 Kleinkauf N, Verweij P, Arendrup M et al. European Centre for Disease Prevention and Control. Risk assessment on the impact of environmental usage of triazoles on the development and spread of resistance to medical triazoles in Aspergillus species. ECDC, Stockholm, Germany (2013).

13 Hamprecht A, Buchheidt D, Vehreschild JJ et al. Azole-resistant invasive aspergillosis in a patient with acute myeloid leukaemia in Germany. Euro. Surveill. 17(36), 20262 (2012).

14 Seyedmousavi S, Bruüggemann RJ, Melchers WJ et al. Posaconazole prophylaxis in experimental azole resistant invasive pulmonary aspergillosis. Presented at: 53rd Interscience Conference on Antimicrobial Agents and Chemotherapy (ICAAC). Denver, Colorado, USA, 10-13 September 2013.

15 Seyedmousavi S, Bruüggemann RJ, Melchers WJ et al. Intrapulmonary posaconazole penetration at the infection site in an immunosuppressed murine model of invasive pulmonary aspergillosis receiving oral prophylactic regimens. Antimicrob. Agents Chemother. 58(5), 2964-2967 (2014).

16 Auberger J, Lass-Flöri C, Aigner $\mathrm{M}$ et al. Invasive fungal breakthrough infections, fungal colonization and emergence of resistant strains in high-risk patients receiving antifungal prophylaxis with posaconazole: real-life data from a single-centre institutional retrospective observational study. J. Antimicrob. Chemother. 67(9), 2268-2273 (2012).

17 Strasfeld L, Espinosa-Aguilar L, Gajewski JL et al. Emergence of Cunninghamella as a pathogenic invasive mold infection in allogeneic transplant recipients. Clin. Lymphoma Myeloma Leuk. 13(5), 622-628 (2013).

18 Park B, Pappas PG, Wannemuehler KA et al. Invasive non-Aspergillus mold infections in transplant recipients, United States, 2001-2006. Emerg. Infect. Dis. 17(10), 1855-1864 (2011).

19 Legouge C, Caillot D, Chrétien ML et al. The reversed halo sign: pathognomonic pattern of pulmonary mucormycosis in leukemic patients with neutropenia? Clin. Infect. Dis. 58(5), 672-678 (2014). 
Hammond SP, Bialek R, Milner DA et al. Molecular methods to improve diagnosis and identification of mucormycosis. J. Clin. Microbiol. 49(6), 2151-2153 (2011).

21 Skiada A, Pagano L, Groll A et al. Zygomycosis in Europe: analysis of 230 cases accrued by the registry of the European
Confederation of Medical Mycology (ECMM) Working Group on Zygomycosis between 2005 and 2007. Clin. Microbiol. Infect. 17(12), 1859-1867 (2011).

22 Chamilos G, Lewis RE, Kontoyiannis DP. Delaying amphotericin B-based frontline therapy significantly increases mortality among patients with hematologic malignancy who have zygomycosis. Clin. Infect. Dis. 47(4), 503-509 (2008).

23 Herbrecht R, Tissot F, Agrawal S et al. 2013-update of the ECIL Guidelines for Antifungal Therapy in Leukemia and HSCT Patients (ECIL-5). Presented at: 5th European Conference on Infections in Leukaemia. Sophia Antipolis, France, 20-21 September 2013. 